Notre Dame Journal of Formal Logic

Volume 33, Number 2, Spring 1992

\title{
End Extensions of Models of Arithmetic
}

\author{
JAMES H. SCHMERL
}

\begin{abstract}
A concise proof is presented of Wilkie's Theorem that for every model of Peano Arithmetic there is a diophantine equation having no solution in that model but having a solution in some end extension of that model.
\end{abstract}

Kaufmann (in [2]) observed that the Completeness Theorem formalized in Peano Arithmetic can be used to give an alternate proof of the MacDowellSpecker Theorem on elementary end extensions of models of PA. (See Theorem 3 for an outline of his proof.) Rabin [4] proved that every model $\mathrm{OM}$ of PA has an elementarily equivalent extension which solves a Diophantine equation having coefficients in M but having no solution in M. Gaifman [1] asked whether Rabin's Theorem could be improved by requiring that the extension be an end extension. By Matijasevič's solution to Hilbert's Tenth Problem, which was unavailable to Rabin, Gaifman's question is equivalent to asking whether every model of PA has an elementarily equivalent end extension which is not a $\Sigma_{1^{-}}$ extension. After several partial results had been obtained (Manevitz [3], Wilkie [6]), Wilkie [7] proved a comprehensive theorem which yielded an affirmative answer to Gaifman's question. His proof relied heavily on his previously obtained affirmative answer for countable models. In this note we will give a rather quick and direct proof of Wilkie's theorem along the lines of Kaufmann's proof of the MacDowell-Specker Theorem.

Notation and terminology will be that standardly used in the Peano Arithmetic literature. The language $L$ of PA is finite. For $\mathrm{OM}$ an $L$-structure, $L(\mathrm{M})$ is $L$ augmented by constant symbols for elements of M. Note that $\Sigma_{n}$ and $\Pi_{n}$ are sets of $L$-formulas (or $L(\mathrm{M})$-formulas) which have a certain syntactic form. For an $L$-structure $\mathrm{N}$, we let $D(\mathrm{CM})$, the elementary diagram of $\mathrm{dM}$, be the set of $L(\mathrm{M})$-sentences true in $\mathrm{OM}$. We write $\mathrm{oM}<_{n} \mathrm{~N}$ to mean that $\mathrm{N}$ is a $\Sigma_{n}$-elementary extension of $\mathrm{cM}$ or, equivalently, $D(\mathrm{cM}) \cap \Pi_{n} \subseteq D(\mathrm{~N})$. We let $\operatorname{SSy}(\mathrm{cM})$ be the standard system of $\mathrm{OM}$; and for a complete theory $T \supseteq \mathrm{PA}$, we let $\operatorname{Rep}(T)$ be the standard system of its minimal model.

Given a model $\mathrm{OM}$ of PA and an $L$-structure $\mathrm{N}$, we say that $\mathrm{N}$ is internal to 
$\mathcal{M}$ if there is a subset $D \subseteq M$ which is definable in $\mathrm{OM}$ such that $\mathrm{OM}$ " $D$ is a complete and consistent Henkin theory," and the structure that $D$ determines is isomorphic to $\mathcal{N}$. If $\mathcal{N}$ is internal to $\mathrm{N}$ and is a model of a sufficiently strong (but still very weak) finite fragment of PA, then without loss of generality we can take $\mathrm{N}$ to be an end extension of $\mathrm{OM}$.

The following is a version of Wilkie's theorem.

Theorem 1 Let $\mathrm{OM}$ F PA be nonstandard and let $T \supseteq P A$ be a complete theory. Then $\mathrm{N}$ has an end extension $\mathrm{N}$ such that $\mathrm{N} \vDash T$ iff the following two conditions hold:

(1) $\operatorname{Rep}(T) \subseteq \operatorname{SSy}(\mathrm{M})$;

(2) $T \cap \Pi_{1} \subseteq T h(\mathcal{M})$.

Proof: Suppose $\mathcal{O} \subseteq{ }^{\text {end }} \mathcal{N}$ and $\mathcal{N} \vDash T$. Clearly, $\operatorname{Rep}(T) \subseteq \operatorname{SSy}(\mathcal{N})=\operatorname{SSy}(\mathcal{M})$, so that (1) holds. Since $\Sigma_{1}$ sentences persist under end extensions, (2) also holds.

Now suppose (1) and (2) hold. It follows from the Reflection Principle and (2) that $\mathcal{M} \vDash \operatorname{Con}(\ulcorner\sigma\urcorner)$ for each $\sigma \in T$. Then using (2) and (1), overspill, and the Completeness Theorem formalized in $\mathrm{M}$, we can obtain a model

$$
\mathcal{N}_{0} \vDash T \cap \Pi_{1}
$$

which is internal to $\mathcal{M}$ such that $\mathcal{O M} \subseteq{ }^{\text {end }} \mathcal{N}_{0}$. We proceed by constructing a sequence $\mathcal{N}_{0}, \mathcal{N}_{1}, \mathcal{N}_{2}, \ldots$ of structures, each one of which is internal to $\mathrm{M}$. Each $\mathcal{N}_{i+1}$ should be such that

$$
\mathcal{N}_{i+1} \vDash T \cap \Pi_{i+2}
$$

and

$$
\mathcal{N}_{i}<_{i} \mathcal{N}_{i+1} .
$$

The existence of each of the $\mathcal{N}_{i+1}$ is easily confirmed by a diagram argument followed by an application of the Completeness Theorem, both taking place inside of $\mathcal{O}$. Clearly, $\mathcal{N}=\bigcup_{i} \mathcal{N}_{i}$ is an end extension of $\mathcal{O M}$ and $\mathcal{N} \vDash T$.

In the proof of the next theorem we will use Theorem 1.

Theorem 2 (Wilkie) For each nonstandard on there is $\mathcal{N} \equiv \mathcal{M}$ such that $\mathrm{M} \subseteq{ }^{\text {end }} \mathcal{N}$ but $\mathcal{N}$ is not a $\Sigma_{1}$-extension of $\mathrm{M}$.

Proof: It is enough to find such an $\mathcal{N}$, with $\operatorname{Th}(\mathcal{N}) \cap \Sigma_{1} \subseteq$ Th( $(\mathcal{M})$ instead of $\mathcal{N} \equiv \mathcal{N}$, because then Theorem 1 can be applied to it to get the desired model.

Let $\varphi(x)$ be a usual $\Sigma_{1}$ formula defining a simple set as, for example, in Theorem 8.II of Rogers [5]. Thus $\mathcal{M} \vDash \forall x \exists y(\neg \varphi(y) \wedge x \leq y \leq 2 x)$, and for any $\Sigma_{1}$ formula $\psi(x)$ either oN $\vDash \exists x(\varphi(x) \wedge \psi(x))$ or else there is $n<\omega$ such that $\mathcal{M} \vDash \forall x(\psi(x) \rightarrow x \leq n)$. Let $a \in \mathrm{M}$ be nonstandard such that $\mathcal{M} \vDash \neg \varphi(a)$. We will obtain $\mathcal{N}$ such that $\mathcal{N} \vDash \varphi(a)$.

Consider some $\sigma \in \operatorname{Th}(\mathcal{M}) \cap \Pi_{1}$, and let $\psi(x)$ be the $\Sigma_{1}$ formula

$$
\left(\exists \theta \in D(\mathcal{M}) \cap \Pi_{0}\right)[\text { length }(\theta) \leq \text { length }(\sigma) \wedge \neg \operatorname{Con}(\ulcorner\sigma \wedge \theta \wedge \varphi(\dot{x})\urcorner)] .
$$

Clearly, it suffices to prove $\mathrm{M} \vDash \neg \psi(a)$. By the Reflection Principle, $\mathrm{c} \vDash \vDash$ $\forall x(\varphi(x) \rightarrow \neg \psi(x))$. Therefore $\mathcal{M} \vDash \forall x(\psi(x) \rightarrow x \leq n)$ for some $n<\omega$. Since $a$ is nonstandard, $\mathrm{ON} \vDash \neg \psi(a)$. 
The previous theorems have hierarchal versions which we now only state, but for which similar proofs exist. For the remainder of this note fix some $k<\omega$.

Theorem 1.k Let $\mathrm{OM}$ ₹ PA be nonstandard and let $T \supseteq \mathrm{PA}$ be a complete theory. Then $\mathrm{O}$ has an end $\Sigma_{k}$-extension $\mathrm{N}$ such that $\mathrm{N} F T$ iff the following two conditions hold:

(1) $\operatorname{Rep}(T) \subseteq \operatorname{SSy}(\mathrm{M})$;

(2) $T \cap \Pi_{k+1} \subseteq \operatorname{Th}(\mathrm{cM})$.

Theorem 2.k For each nonstandard on there is $\mathrm{N} \equiv \mathrm{cM}$ such that $\mathrm{N}<_{k}^{\text {end }} \mathrm{N}$ but $\mathcal{N}$ is not a $\Sigma_{k+1}$-extension of $\mathrm{M}$.

For completeness, we end with a sketch of Kaufmann's proof of the MacDowell-Specker Theorem.

Theorem 3 (MacDowell-Specker) Every model of PA has a proper elementary end extension.

Proof: Let $M$ F PA. Much as we did in the proof of Theorem 1, we construct a sequence $\mathcal{N}_{0}, \mathcal{N}_{1}, \mathcal{N}_{2}, \ldots$ of structures, each of which is internal to $\mathrm{M}$ such that

$$
\mathcal{N}_{i} \prec_{i+1} \mathcal{N}_{i+1}
$$

and

$$
\mathcal{M}<i+2 \text { end } \mathcal{N}_{i} \text {. }
$$

Then $\mathcal{N}=\bigcup_{i} \mathcal{N}_{i}$ is an elementary end extension of $\mathcal{c}$. Note that $\mathcal{N}_{0}$ is internal to $\mathrm{NM}$, so by Tarski's theorem on the undefinability of truth, $\operatorname{Th}\left(\mathcal{N} \mathcal{N}_{0}\right) \neq \operatorname{Th}(\mathcal{C M})$, and therefore $\mathcal{N}_{0}$ (and thus also $\mathcal{N}$ ) is a proper extension of $\mathcal{N}$.

\section{REFERENCES}

[1] Gaifman, H., "A note on models and submodels of arithmetic," pp. 128-144 in Conference in Mathematical Logic, London, 1970, Lecture Notes in Mathematics 255, Springer-Verlag, Berlin, 1972.

[2] Kaufmann, M., "Model theory for arithmetic and for set theory: a brief comparative survey," presented at the meeting of the Association for Symbolic Logic, University of Notre Dame, April 1984.

[3] Manevitz, L. M., "Internal end-extensions of Peano Arithmetic and a problem of Gaifman," Journal of the London Mathematical Society, vol. 13 (1976), pp. 80-82.

[4] Rabin, M. O., "Diophantine equations and non-standard models of arithmetic," pp. 151-158 in Logic, Methodology and Philosophy of Science, Proceedings of the 1960 International Congress, Stanford University Press, Palo Alto, 1962.

[5] Rogers, H., Jr., Theory of Recursive Functions and Effective Computability, McGraw-Hill, New York, 1967.

[6] Wilkie, A. J., "On models of arithmetic-Answers to two problems raised by $\mathrm{H}$. Gaifman," The Journal of Symbolic Logic, vol. 40 (1975), pp. 41-47. 
[7] Wilkie, A. J., "On the theories of end-extensions of models of arithmetic," pp. 305309 in Set Theory and Hierarchy Theory V, Lecture Notes in Mathematics 619, Springer-Verlag, Berlin, 1977.

Department of Mathematics

University of Connecticut

Storrs, Connecticut 06269 